\title{
Robust visual tracking via efficient manifold ranking with low-dimensional compressive features
}

\author{
Tao Zhou ${ }^{\mathrm{a}}$, Xiangjian $\mathrm{He}^{\mathrm{b}}$, Kai Xie ${ }^{\mathrm{a}}$, Keren Fu ${ }^{\mathrm{a}}$, Junhao Zhang ${ }^{\mathrm{a}}$, Jie Yang,* \\ ${ }^{a}$ Institute of Image Processing and Pattern Recognition, Shanghai Jiao Tong University, \\ China \\ ${ }^{b}$ Faculty of Engineering and Information Technology, University of Technology, Sydney, \\ Australia
}

\begin{abstract}
In this paper, a novel and robust tracking method based on efficient manifold ranking is proposed. For tracking, tracked results are taken as labeled nodes while candidate samples are taken as unlabeled nodes. The goal of tracking is to search the unlabeled sample that is the most relevant to the existing labeled nodes. Therefore, visual tracking is regarded as a ranking problem in which the relevance between an object appearance model and candidate samples is predicted by the manifold ranking algorithm. Due to the outstanding ability of the manifold ranking algorithm in discovering the underlying geometrical structure of a given image database, our tracker is more robust to overcome tracking drift. Meanwhile, we adopt non-adaptive random projections to preserve the structure of original image space, and a very sparse measurement matrix is used to efficiently extract low-dimensional compressive features for object representation. Furthermore, spatial context is used to improve the robustness to appearance variations. Experimental results on some challenging video sequences show the proposed algorithm outperforms seven state-of-the-art methods in terms of accuracy and robustness.
\end{abstract}

Keywords: Visual tracking; Appearance model; Manifold ranking; Random projections; Low-dimensional compressive features; Spatial context

\footnotetext{
*Corresponding author. Tel. +86 2134204033

Email address: jieyang@sjtu.edu.cn (Jie Yang)
}

Preprint submitted to Pattern recognition

November 28, 2014 


\section{Introduction}

Visual tracking is a long standing research topic due to its wide range of applications such as behavior analysis, activity recognition, video surveillance, and human-computer interaction [1, 2]. Although it has had a significant progress 5 in the past decades, developing an efficient and robust tracking algorithm is still a challenging problem due to numerous factors such as partial occlusion, illumination variation, pose change, abrupt motion, and background clutter. These factors can lead to wrong association, and result in drift and even failure in tracking.

The main tracking algorithms can be categorized two classes: generative [3, 4, 5, 6, and discriminative methods [7, 8, 9, 10, 11, 12, 13.

Generative methods focus on searching for the regions which are the most similar to the tracked targets with minimal reconstruction errors of tracking. Adaptive models including the WSL tracker [3] and IVT method [14] have been proposed to handle appearance variation. Recently, sparse representation methods have been used to represent an object by a set of trivial target templates and trivial templates [6, 15] to deal with partial occlusion, pose variation and so on. Therefore, it is critical to construct an effective appearance model in order to handle various challenging factors. Furthermore, generative methods discard useful information surrounding target regions that can be exploited to better separate objects from backgrounds.

Discriminative methods treat tracking as a classification problem that distinguishes the tracked targets from the surrounding backgrounds. A tracking technique called tracking by detection has been shown to have promising results

25 in real-time. This approach trains a discriminative classifier online to separate an object from its background. Collins et al. [7] selected discriminative features online to improve the tracking performance. Boosting method has been used for object tracking through combining weak classifiers to establish a strong classifier to select discriminative features, and some online boosting feature s- 
al. 9] proposed a novel online MIL algorithm for object tracking that achieves superior results with real-time performance. An efficient tracking algorithm based on compressive sensing theories was proposed by Zhang et al. [10. It uses low dimensional features randomly extracted from high dimensional multiscale image features in the foreground and background, and it achieves better tracking performance than other methods in terms of robustness and speed. Moreover, although some efficient feature extraction techniques have been proposed for visual tracking $[8,10,12$, there often exist a large number of samples from which features need to be extracted for classification, thereby entailing computationally expensive operations [9].

The above tracking methods have shown promising performance. However, they have some shortcomings. Firstly, although the goal of a generative method is to learn an object appearance model, an effective searching algorithm and measuring method to match candidate samples to an object model are difficult

45 to obtain. Secondly, background varies broadly during a tracking process, so it is difficult to achieve the aim of a discriminative method to distinguish a target region from a complicated background when the target looks similar to its background. Therefore, it is very difficult to construct a discriminative object representation. Thirdly, feature selection is of crucial importance for generating an effective appearance model. However, approaches using a large amount of features make the computational load very heavy. Therefore, the computational complexity of tracking methods is rather high, , and this limit the real-time applications of these methods.

Graph-based ranking algorithms have been widely applied to information retrieval and have proved to have excellent performance and feasibility on a variety of data types [17, 18, 19]. The manifold ranking algorithm first constructs a weighted graph by considering each data node as a vertex. The ranking score of the query is iteratively propagated to nearby node via the weighted graph. Finally nodes will be ranked according to the ranking scores, in which a larger ${ }_{60}$ score indicates higher relevance. In this paper, we develop a novel and robust tracking method based on manifold ranking, which regards tracking as a rank- 
ing problem. As shown in Figure 1, we mark the tracked results as labeled nodes, while candidate samples are regarded as unlabeled nodes. The tracking objective is to estimate the corresponding likelihood that is determined by the relevance between the queries and all candidate samples. We use a manifold structure to measure the relevance between a model and samples, and in our method low-dimensional compressive features can efficiently compress features of foreground objects and their background. Experimental results on some challenging video sequences are demonstrated to show the effectiveness and robustness of the proposed model and algorithm in comparison with seven state-of the-art tracking methods.

The main contributions of this paper are as follows.

1. A novel visual tracking method based on graph-manifold ranking is proposed.

2. An efficient manifold ranking algorithm is adopted, it can reconstruct graph efficiently in each tracking round and reduce the computation complexity.

3. Low-dimensional compressive features of an image are extracted by a very sparse measurement matrix for object representation. This matrix preserves the structure of the image and discriminates objects from their cluttered background effectively.

4. Our method exploits both temporal and spatial context information, and it is robust to appearance variations caused by abrupt motion, occlusion and background clutters.

5. Experimental results show that the proposed algorithm outperforms seven state-of-the-art methods in terms of accuracy and robustness.

This is is an extension of our paper showing preliminary results in [20]. The rest of this paper is organized as follows. Graph-manifold ranking algorithm is described in Section 2.1. Details of our proposed method based on an efficient 90 manifold ranking with low-dimensional compressive features are demonstrated in Section 3. The efficient manifold ranking algorithm is described in Section 
2.2. Experimental results are shown and analyzed in Section 4. The conclusion is presented in Section 5 .

\section{Preliminaries}

\subsection{Graph-based manifold ranking}

Manifold Ranking (MR), a graph-based ranking algorithm, has been widely applied in information retrieval and shown to have excellent performance and feasibility on a variety of data types [17, 18. The manifold ranking method is described as follows: given a query node, the remaining unlabeled nodes are ranked based on their relevance to the given query. The goal is to learn a ranking function to define the relevance between unlabeled nodes and this query [18, 19].

In [19, 21, a ranking method that exploits the intrinsic manifold structure of data for graph labelling is proposed. Given a data set $X=\left\{x_{1}, x_{2}, \cdots, x_{n}\right\} \in$ $\Re^{m \times n}$, where $m$ is the dimension of feature vector and $n$ is the number of the data set, some data points are labelled queries and the rest need to be ranked according to their relevance to the queries. Let $f: X \rightarrow \Re^{n}$ denote a ranking function which assigns a ranking value $r_{i}$ to each point $x_{i}$, and $r$ is a column vector defined by $r=\left[r_{1}, r_{2}, \cdots, r_{n}\right]^{T}$. Let $y=\left[y_{1}, y_{2}, \cdots, y_{n}\right]^{T}$ denote an indication vector, in which $y_{i}=1$ if $x_{i}$ is a query, and $y_{i}=0$ otherwise. Suppose all data points represent a graph $G=(V, E)$, where $V$ represents vertex set, and edges $E$ are weighted by an affinity matrix $W=\left[w_{i j}\right]_{n \times n}$. The strength of edge reflects the similarity between two vertices. To find the optimal ranking of queries, the cost function associated with $r$ is defined as follows.

$$
O(r)=\frac{1}{2}\left(\sum_{i, j=1}^{n}\left\|\frac{1}{\sqrt{D_{i i}}} r_{i}-\frac{1}{\sqrt{D_{j j}}} r_{j}\right\|^{2}+\mu \sum_{i=1}^{n}\left\|r_{i}-r_{j}\right\|^{2}\right)
$$

where $\mu>0$ controls the balance of the smoothness constraint (the first term) and the fitting constraint (the second term), $D$ is a diagonal matrix with the element $D_{i i}=\sum_{j=1}^{n} w_{i j}$, for $i, j=1,2, \cdots, N$. To minimize the cost function, we can obtain the closed form solution as:

$$
r^{*}=(I-\alpha S)^{-1} y
$$


where $I$ is an identity matrix, $\alpha=\frac{1}{1+\mu}$ and $S=D^{-\frac{1}{2}} W D^{-\frac{1}{2}}$. Then, we use the

iteration scheme to solve the following optimal problem:

$$
r(t+1)=\alpha S(r(t)+(1-\alpha) y)
$$

where $\alpha$ is control parameter, which balances each points information from its neighbors and and the original information.

\subsection{Efficient manifold ranking algorithm}

In order to efficiently reconstruct graph, we use efficient manifold ranking algorithm [19] to compute the ranking score. First, we briefly introduce how to use anchor graph to model the data. Given a data set $X=\left\{x_{1}, x_{2}, \cdots, x_{n}\right\} \in$ $\Re^{m \times n}, U=\left\{u_{1}, u_{2}, \cdots, u_{d}\right\} \in \Re^{m \times d}$ indicates a set of anchors sharing the same space with the data set. Then, we define a real value function $r: X \rightarrow R$, which assigns a semantic label for each point in $X$. The aim is to find a weight matrix that measures relevance between data points $r: X \rightarrow R$ and anchors in $U$. We obtain $r(x)$ for each point by a weighted average of these labels on anchors as follows:

$$
r\left(x_{i}\right)=\sum_{k=1}^{d} z_{k i} r\left(u_{k}\right), i=1,2, \cdots, n
$$

where $\sum_{k=1}^{d} z_{k i}=1$ and $z_{k i}>0$, in which $z_{k i}$ represents the weight between point $x_{i}$ and an anchor $u_{k}$. The weights can be obtained by Nadaraya-Watson kernel regression to increase smoothness. The graph construction process and the means to get the anchors can be found in detailed [19].

We use a new approach to represent the adjacency matrix $W$. The weight matrix $Z \in \Re^{d * n}$ can be viewed as a d-dimensional representation of the data $X \in \Re^{m * n}$, in which $d$ is the number of anchor points. It means that data points can be presented in a new space to replace the original feature space. We set the adjacency matrix as follows:

$$
W=Z^{T} Z
$$


where $W_{i j}>0$ if two points are correlative and they will share at least one common anchor point, otherwise $W_{i j}=0$. The new adjacency matrix is useful to explore relevance among data points. According to $W=Z^{T} Z$, equation (2) can be rewritten as follows:

$$
r^{*}=\left(T_{1}-\alpha H^{T} H\right)^{-1} y=\left(I_{1}-H^{T}\left(H H^{T}-\frac{1}{\alpha} I_{2}\right)^{-1} H\right) y
$$

where $H=Z D^{-1}$ and $S=H^{T} H, I_{1}$ and $I_{2}$ are the identity matrices. By equation(6), the inversion computation part have changed form a matrix $n \times n$ to a matrix $d \times d$. Therefore, the change can efficiently reduce computation load for $d \ll n$. We can also note that the matrix $D$ is computed without using the adjacency matrix $W$. In equation (6),

$$
D_{i i}=\sum_{j=1}^{n} w_{i j}=\sum_{j=1}^{n} z_{i}^{T} z_{j}=z_{i}^{T} v
$$

where $z_{i}$ is the $i$ th column of $Z$, and $v=\sum_{j=1}^{n} z_{j}$. Thus, we compute the matrix $D$ without using the adjacency matrix $W$. By the equation (6), we multiply a matrix to avoid the matrix - matrix multiplication every time. Therefore, the efficient manifold ranking algorithm has a complexity $O\left(d n+d^{3}\right)$. Due to a low complexity for computing the ranking function $r^{*}$, we can reconstruct graph in each tracking round efficiently.

\subsection{Low-dimensional compressive features}

The Haar-like features have been widely used for object representation and appearance modeling. They are typically designed for different tasks such as object detection, object tracking and et al. [9, 10, 22. However, Harr-like features require high computational loads for feature extraction in training and tracking phases. Recently, Babenko et al. 9] adopted the generalized Haar-like features where each one is a linear combination of randomly generated rectangle features, and used online boosting to select a small set of them for object tracking. In our tracking framework, we use the low-dimensional compressive features proposed by Zhang et al. [10] for the appearance modelling. A large 
set of Haar-like features is significantly compressed using a very sparse measurement matrix. Object representation using the compressed features preserves the object structure represented in the original feature space. and these features in the compressed domain can be applied efficiently.

Given a random matrix $R \in \Re^{n * m}$ that projects a high-dimensional image feature $x \in \Re^{m}$ to a low-dimensional feature $v \in \Re^{n}$ :

$$
v=R x
$$

where $n \ll m$. Ideally, the matrix $R$ can provide a stable embedding to preserve the distance between all pairs of original signals, in other words, lowerdimensional features can recover original high-dimensional information. JohnsonLindenstrauss lemma [23] states that with high probability the distances between the points in a vector space are preserved if they are projected onto a randomly selected subspace with suitably high dimensions. Therefore, if the random matrix $R$ in equation(8) meets Johnson-Lindenstrauss lemma, we can reconstruct the original data $x$ with minimum error from low-dimensional data $v$ with high probability when $x$ is compressive such as video or image. Thus, a very sparse matrix is used for extracting compressive features, and it only satisfies Johnson-Lindenstrauss lemma and the real-time applications.

A typical measurement matrix satisfying the restricted isometry property is the random Gaussion matrix $R \in \Re^{n * m}, r_{i j} \sim N(0,1)$, so a very sparse random measurement matrix is defined as:

$$
r_{i j}=\sqrt{s} \times\left\{\begin{aligned}
1 & \text { with probability } 1 / 2 s \\
0 & \text { with probability } 1-1 / s \\
-1 & \text { with probability } 1 / 2 s
\end{aligned}\right.
$$

In order to satisfy Johnson-Lindenstrauss lemma, the measurement matrix should be with $s=2$ or $s=3[23$. We can note the measurement matrix is very easy to compute which requires only a uniform random generator. In order to enhance separability, distinguish ability and adaptability at fixed scale, samples from this fixed scale are convolving with a set of rectangle filters at multiple 
scales. Each rectangular filter at a scale is defined

$$
h_{p, q}(x, y)= \begin{cases}1 & 1 \leq x \leq p, 1 \leq y \leq q \\ 0 & \text { otherwise }\end{cases}
$$

where $\left(x_{i}, y_{i}\right)$ is the coordinates of the upper left corner in the rectangular filter, $p$ and $q$ are the width and height of the rectangle filter respectively.

$S_{u}=\left\{x_{1}^{s+1}, x_{2}^{s+1}, \cdots, x_{i}^{s+1}\right\}, i=1,2, \cdots, N$, and the corresponding graph is taken as $G_{u}$. Thirdly, the candidate $G_{u}$ is combined with $G_{m}$ to construct a graph $G=G_{m} \cup G_{u}$, in which the label $y_{i}=1$ if a node point is in $G_{m}$, and 


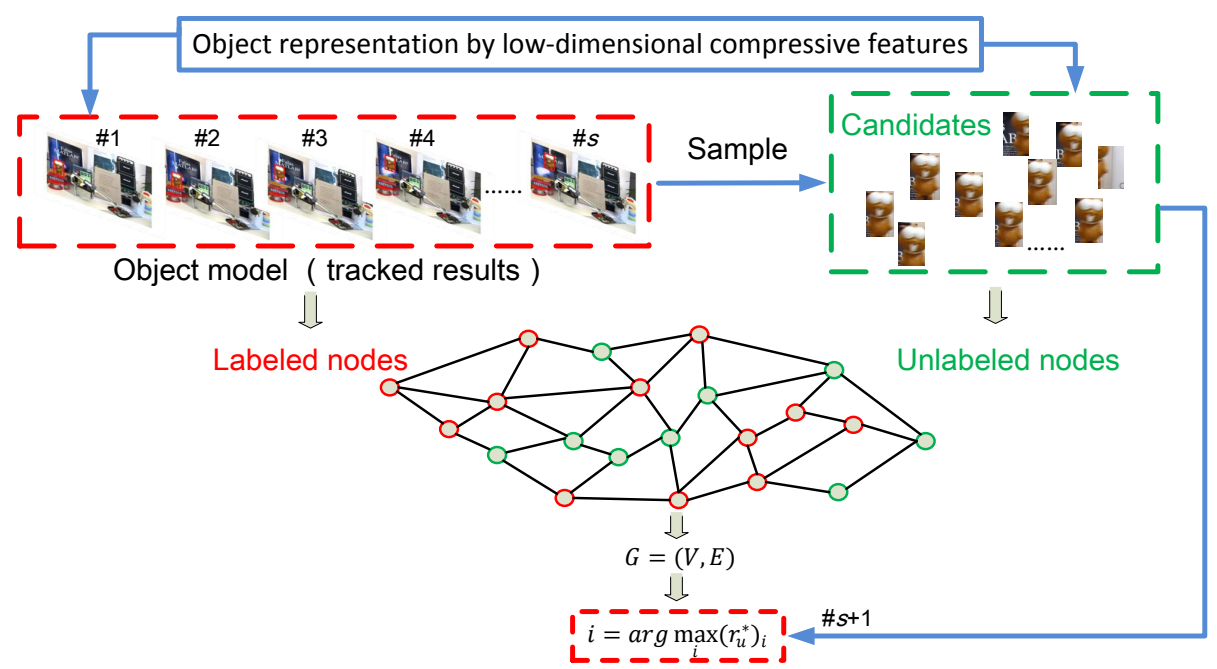

Figure 1: Basic flow of our tracking algorithm. A graph is established combining labeled nodes (tracked results) and unlabeled nodes (candidate samples), and ranking scores represent the relevance between the object model and candidate samples.

$y_{i}=0$ if a node point is in $G_{u}$. The ranking score vector $r^{*}=\left[r_{m}^{*} ; r_{u}^{*}\right]$ can be obtained by manifold ranking algorithm, where $r_{m}^{*}$ is corresponding to $G_{m}$ while $r_{u}^{*}$ is corresponding to $G_{u}$. Then, the tracking result is added into $S_{m}$, while the other candidate samples are deleted. This procedure continues to sample candidates and construct a new graph to obtain the largest ranking score as the tracking result until the end of the image sequence.

\subsection{Graph construction}

We construct a graph $G=(V, E)$ in shown Figure 1, where $V$ is vertex set and $E$ is edge set. As an object has similar appearance in continuous frames, each node is connected to those nodes neighboring it to exploit the continuous relationship. In our work, tracked results are taken as labeled nodes while candidate samples are taken as unlabeled nodes, so $w_{i j}$ reflects how closely the $i$-th node is linked to the $j$-th node. Meanwhile, $W \in \Re^{n * m}$ denotes the adjacency matrix with element $w_{i j}$ also indicating the weight of the edge between points $i$ and $j$, for $i, j=1,2, \cdots, n$. If there exists an edge linking the $i$-th node 
and the $j$-th node, the weight between the two nodes can be defined by

$$
w_{i j}=e^{-\frac{\left\|c_{i}-c_{j}\right\|^{2}}{2 \sigma^{2}}}
$$

235 space respectively, $\sigma$ is a constant that controls the strength of the weight. By ranking all nodes on the construction graph, we can obtain a nonzero relevance value for any nodes on the graph.

\subsection{Appearance model updating process}

a CT tracker, and then to obtain the location of the $t+1$ frame by manifold ranking algorithm. There exists an obvious problem that the size of $S_{m}$ will be very large if all tracked results are added into the appearance model in each tracking round, so the computation complexity will be very heavy. In addition, next frame, we need to update the appearance model firstly. We compute the average ranking score of $r_{m}^{*}$;

$$
\mu_{r_{m}^{*}}=\sum_{i=1}^{t}\left(r_{m}^{*}\right)_{i}
$$

where $\left(r_{m}^{*}\right)_{i}$ represents the score of the $i$-th node in $S_{m}$. Then, we compute the displacement error $e_{i}$ between the score of each node in $S_{m}$ and the average score:

$$
e_{i}=\left\|\left(r_{m}^{*}\right)_{i}-\mu_{r_{m}^{*}}\right\|^{2}
$$

We delete the node that has the largest displacement error, and then add the current tracking result $x_{t+1}^{*}$ into $S_{m}$. Thus, the number of $S_{m}$ will be $t$ constantly. It is worth noting that the average ranking score computed from tracked results alleviates the noise effects. 
Algorithm 1. The proposed tracking method

Input: Video frame $f=1: F$

1. The first $t$ frames are tracked by a CT tracker to construct an object appearance model set

$S_{m}=\left\{x_{1}^{*}, x_{2}^{*}, \cdots, x_{i}^{*}\right\}$

2. for $f=t+1$ to $F$ do

3. Crop out a set of candidate samples as unlabeled set $S_{u}$ by $\left.x^{\beta}=\left\{x: \| l(x)-l_{t}\left(x^{*}\right)\right) \|<\beta\right\}$.

4. if $f==t+1$

5. Construct a graph $G=G_{m} \cup G_{u}$ and support set $S_{s}$.

6. Update model set $S_{m}$.

7. else

8. Construct a graph $G=G_{m} \cup G_{s} \cup G_{u}$.

9. $\quad$ Update model set $S_{m}$ and support set $S_{s}$.

10. end if

11. The $i$-th candidate sample that has the largest in all $r_{u}$ is taken as the object location, the $i$-th sample can be selected by $i=\operatorname{argmax}_{i} r_{u}^{*}, i=1,2, \cdots, U$, where $U$ is the number of candidate samples.

12. end for

Output: Tracking results $\left\{l_{1}\left(x^{*}\right), l_{2}\left(x^{*}\right), \cdots, l_{F}\left(x^{*}\right)\right\}$.

\subsection{Support set construction}

In our method, object appearance model $S_{m}$ only reflects the temporal relationship among consecutive frames, while it can not consider its immediate surrounding background. In the tracking process, the context of a target in an image sequence consists of the spatial context including the local background and the temporal context including all appearances of the targets in the previous frames. As shown in Figure 2 (left), our object appearance model $S_{m}$ repre- 


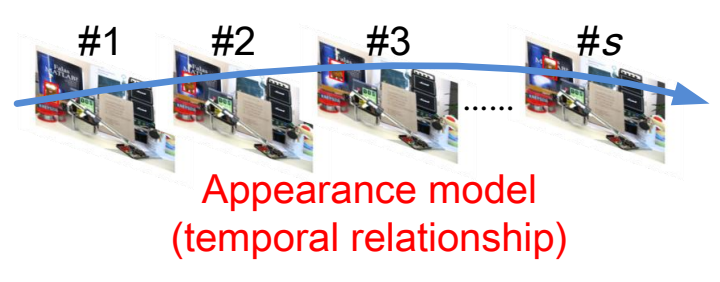

(temporal relationship)

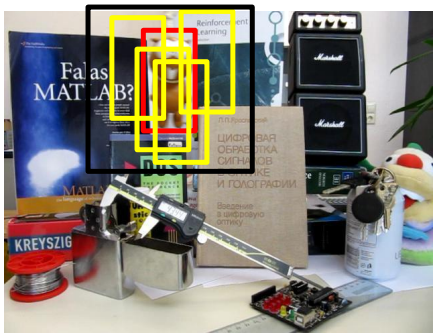

Spatial relationship

Figure 2: Temporal and spatial relationship.

sents the temporal context in the previous frames. In Figure 2 (right), note that the object can be influenced by its surrounding background, and there exists a efficient manifold ranking algorithm (see Section 2.2 , where $r_{m}^{*}, r_{s}^{*}$, and $r_{u}^{*}$ are 


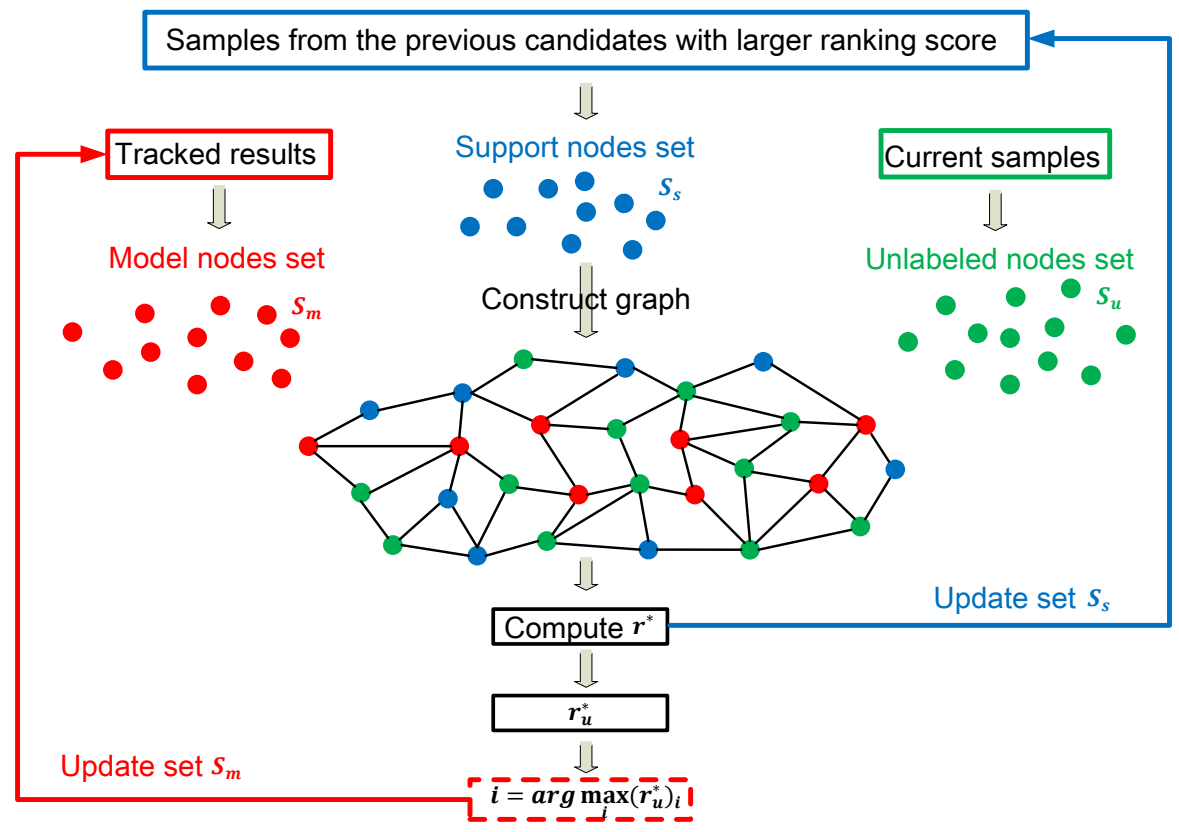

Figure 3: The updating process of appearance model and the construction of support set.

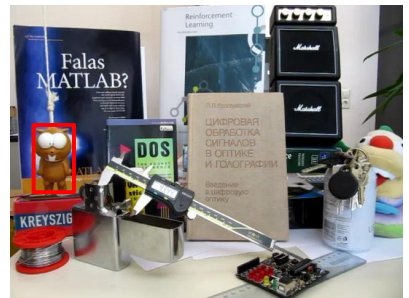

(a) Lemming

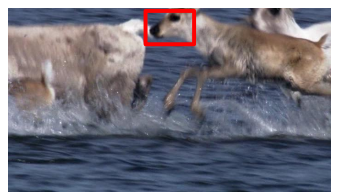

(d) Deer

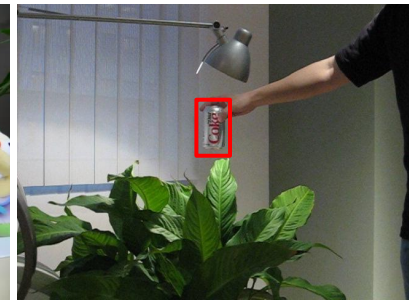

(b) Coke

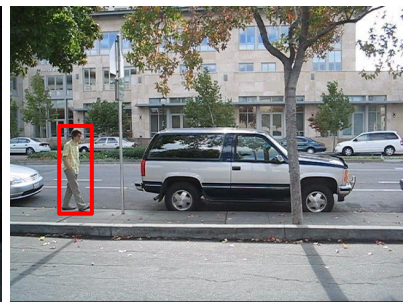

(c) DavidOutdoor

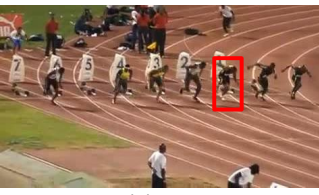

(g) Bolt

Figure 4: The caption of tracking different object in video sequences. 
Table 1: Evaluated video sequences.

\begin{tabular}{|l|l|l|}
\hline Sequences & \#Frames & Challenging Factors \\
\hline Deer & 71 & abrupt motion, background clutter \\
\hline Coke & 291 & abrupt motion, partial occlusion \\
\hline Bolt & 293 & partial occlusion, scale change \\
\hline Stone & 593 & partial occlusion, background clutter \\
\hline Couple & 140 & partial occlusion, abrupt motion, background clutter \\
\hline Lemming & 1336 & partial occlusion, abrupt motion, background clutter \\
\hline DavidIndoor & 252 & partial occlusion, illumination variation \\
\hline
\end{tabular}

corresponding to $G_{m}, G_{s}, G_{u}$ respectively. The tracking scheme is summarized in Algorithm 1. Finally, the target in frame $t+2$ is the sample with the largest component in $r_{u}^{*}$, as the $i$-th sample can be selected from $S_{u}$ and computed by

$$
i=\underset{i}{\operatorname{argmax}} r_{u}^{*}, i=1,2, \cdots, U
$$

where $U$ is the number of candidate samples.

\section{Experimental results and analysis}

\subsection{Experimental setup}

We evaluate the proposed tracking method based on an efficient manifold ranking algorithm and an object representation with low-dimensional features using seven video sequences with impacted factors including abrupt motion, cluttered background, severe occlusion and appearance change (See Table 1). We compare our proposed tracker with seven other state-of-the-art methods including: L1 tracker (L1) 6], real-time compressive tracking (CT) [10, multiple instance learning tracker (MIL) 9], incremental visual tracking (IVT) [14, fragment tracker (Frag) 4], weighted multiple instance learning tracker (WMIL) 24] and locally orderless tracking (LOT) 25]. For fair comparison, we adopt the source or binary codes provided by the authors with tuned parameters for best performance. But for some trackers involving randomness, we repeat the experimental results 5 times on each sequence and obtain the averaged results. 
Table 2: Center location error (CLE). Red fonts indicate the best performance while the blue fonts indicate the second best ones.

\begin{tabular}{lcccccccc}
\hline Sequence & L1 & CT & MIL & IVT & Frag & WMIL & LOT & Ours \\
\hline Coke & 85.3 & 42.0 & 26.9 & 70.5 & 124.8 & 76.9 & 42.5 & 23.9 \\
Bolt & 39.4 & 211.4 & 35.8 & 138.8 & 18.8 & 214.3 & 68.2 & 7.6 \\
Deer & 171.5 & 95.1 & 66.5 & 127.5 & 92.1 & 25.1 & 65.9 & 23.0 \\
Stone & 19.2 & 32.8 & 32.3 & 2.5 & 65.9 & 99.8 & 28.1 & 6.4 \\
Couple & 110.6 & 33.8 & 33.9 & 105.1 & 32.6 & 34.4 & 37.8 & 9.3 \\
Lemming & 184.9 & 26.3 & 25.9 & 93.4 & 149.1 & 96.9 & 19.9 & 24.3 \\
DavidOutdoor & 100.4 & 87.3 & 38.4 & 52.9 & 90.5 & 73.3 & 63.5 & 29.5 \\
\hline Average CLE & 101.6 & 75.5 & 37.1 & 84.4 & 81.9 & 88.7 & 46.6 & 17.7 \\
\hline
\end{tabular}

In our experiments, the parameters are used in our algorithm as follows. The search radius for cropping out candidate samples is set to $\beta=20$, which is related with object motion speed. The dimensionality of the compressive feature is set to 200. The first $t$ frames are tracked by the CT method and $t$ is set to 30 . The number of nodes in support set is set $s=10$. Implemented in MATLAB, our tracking method runs at about 10 frames per second (FPS) to obtain the averaged results on an i3 $3.20 \mathrm{GHz}$ machine with 4 GB RAM.

\subsection{Quantitative analysis}

We perform experiments on seven publicly available standard video sequences. As the ground truth, the center position of a target in a sequence is labeled manually. This ground truth is provided in Wu's work [26]. For quantitative analysis, we use average center location errors as evaluation criteria to compare the performance, and the pixel error in every frame is defined as follow:

$$
C L E=\sqrt{\left(x^{\prime}-x\right)^{2}+\left(y^{\prime}-y\right)^{2}}
$$

where $\left(x^{\prime}, y^{\prime}\right)$ represents the object position obtained by different tracking methods, and $(x, y)$ is the ground truth. The second evaluated metric is the success 
Table 3: Success rate (SR)(\%). Red fonts indicate the best performance while the blue fonts indicate the second best ones.

\begin{tabular}{|c|c|c|c|c|c|c|c|c|}
\hline Sequence & L1 & $\mathrm{CT}$ & MIL & IVT & Frag & WMIL & LOT & Ours \\
\hline Coke & 13.1 & 50.2 & 72.2 & 15.8 & 3.5 & 44.8 & 13.7 & 79.4 \\
\hline Bolt & 27.5 & 4.7 & 44.4 & 3.4 & 54.6 & 3.1 & 17.4 & 81.7 \\
\hline Deer & 3.9 & 14.1 & 21.3 & 11.7 & 7.6 & 83.5 & 35.2 & 85.9 \\
\hline Stone & 29.2 & 35.2 & 32.1 & 65.2 & 15.4 & 8.4 & 27.8 & 65.2 \\
\hline Couple & 12.3 & 67.8 & 71.4 & 10.1 & 64.3 & 65.3 & 69.7 & 92.8 \\
\hline Lemming & 3.9 & 74.8 & 53.5 & 17.8 & 13.4 & 24.4 & 84.5 & 82.1 \\
\hline DavidOutdoor & 27.5 & 22.4 & 64.8 & 41.1 & 19.5 & 29.8 & 31.2 & 72.3 \\
\hline Average SR & 16.8 & 38.5 & 51.4 & 23.9 & 25.5 & 37.1 & 40.0 & 79.9 \\
\hline
\end{tabular}

rate [27, and the score in every frame is defined as follow:

$$
\text { score }=\frac{\operatorname{area}\left(R O I_{T} \cap R O I_{G}\right)}{\operatorname{area}\left(R O I_{T} \cup R O I_{G}\right)}
$$

where $R O I_{T}$ is the tracking bounding box and $R O I_{G}$ is the ground truth bounding box. If the score is larger than 0.5 in one frame, the tracking result is considered as a success. Table 2 reports the center location error, where smaller CLE means more accurate tracking results. In Table 2, each row represents the average center location errors of the eight algorithms testing on a certain video sequence. The number marked with red indicates the best performance in a certain testing squence, and blue indicates the second best. Table 3 reports the success rates, where larger average scores mean more accurate results. From 325 Table 2 and Table 3 , we can see that our method achieves the best or second best performance compared with L1, CT, MIL, WMIL, Frag, IVT and LOT for most of sequences. Moreover, we draw the error curve according to equation 15) for each video sequences (Figure 5). In addition, Figure 6, Figure 7 and Figure 8 show the screen captures for some of the video clips. More details of experiments are analyzed and discussed in the following subsections. 

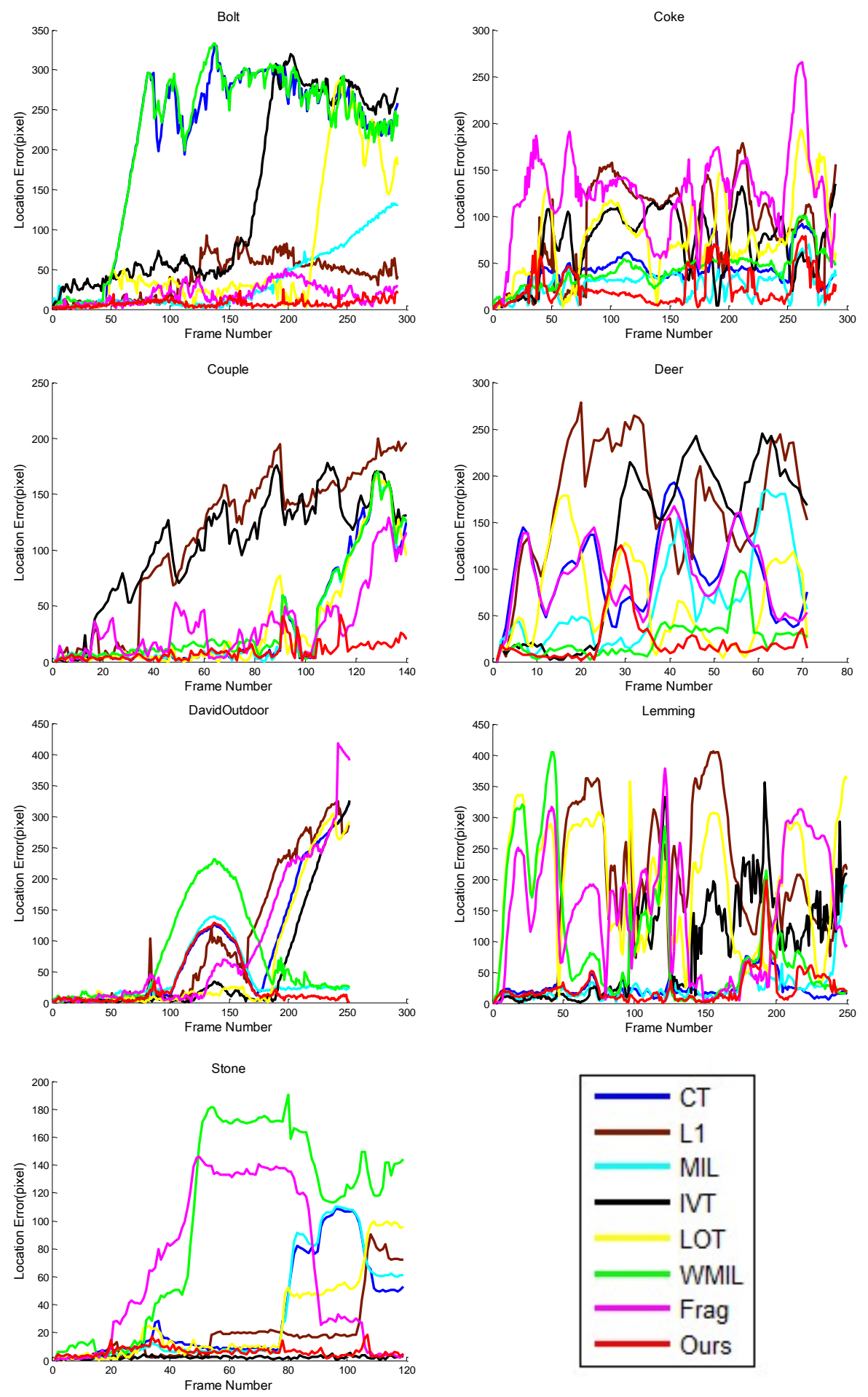

Figure 5: Error plots of all tested sequences for different tracking methods. 

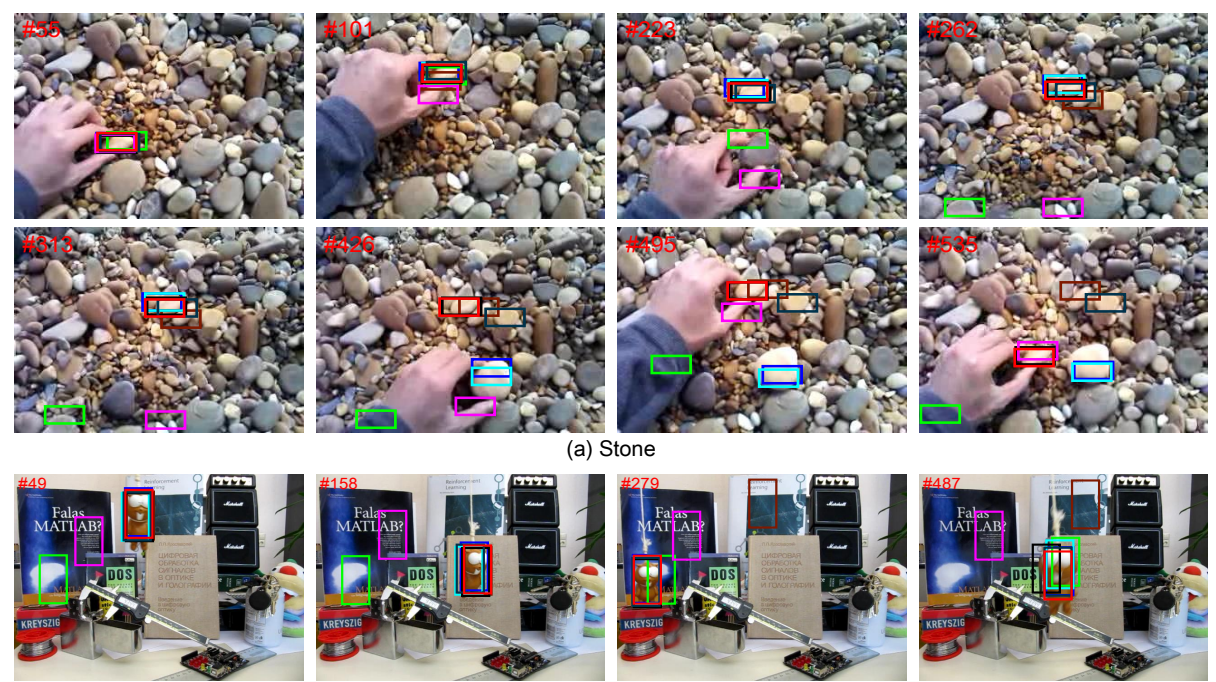

(a) Stone
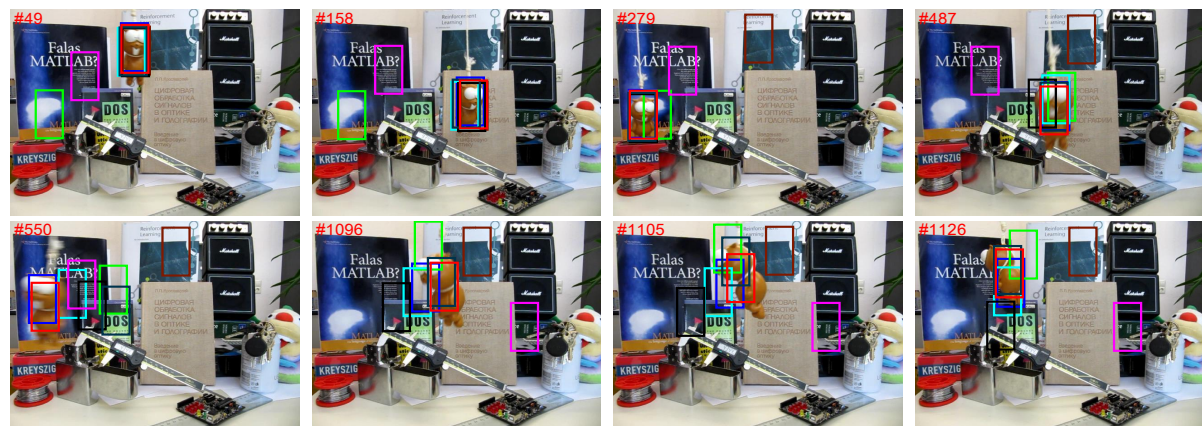

(b) Lemming

- L1 - CT - MIL - IVT - Frag - WMIL - LOT — Ours

Figure 6: Sampled tracking results for tested sequences of (a) Stone and (b) Lemming. 


\subsection{Qualitative analysis}

Partial occlusion: The objects suffer heavy or longtime partial occlusion, scale change, deformation and rotation in sequences Bolt(Figure 8(a)), Lemming (Figure 7(b)), DavidOutdoor(Figure 6(c)), Coke (Figure 6(a))). In the Bolt sequence, Figure 8 (a) demonstrate that our tracking method performs well in terms of position and scale when the objects undergo severe occlusion and deformation at frames \#112, \#157, \#167, while the other methods including the IVT, CT, WMIL and L1 completely fail to track the objects in these frames. This can be attributed to some reasons: (1) we can extract discriminative features by a very sparse matrix to separate an object well from its background, and also object representation with low-dimensional compressive features can preserve the structure of original image space; and (2) the outstanding ability of manifold ranking algorithm is to discover the underlying geometrical structure and the relevance between object appearance and candidate samples. Thus, our tracker is more robust to overcome tracking drift and abrupt motion. In the DavidOutdoor sequence, our method and MIL perform better than other methods at frames \#193, \#206 and \#252. The other methods suffer from sever drift and some of these methods completely fail to track. Furthermore, our method performs more accurately than MIL at frames \#230 and \#252. Thus, our method can handle occlusion and it is not sensitive to partial occlusion since the manifold ranking algorithm can measure the relevance between object appearance and candidate samples. Furthermore, we can also see the advantages of our tracking method in Lemming and Coke sequences (see Figure 7)(b) and Figure 6(a)).

Abrupt motion and blur: The objects in Deer (Figure 8(b)), Coke (Figure 6(a))), Couple (Figure 6(b)) and Lemming (Figure 7(b)) sequences have abrupt motions. It is difficult to predict the location of a tracked object when it undergoes an abrupt motion. As illustrated in Figure 8(b), when an object undergoes an in-plane rotation, all evaluated algorithms except the proposed tracker do not track the object well. We also see that the WMIL method can track the object well except in frames \#43 and \#56. The CT method suffers 

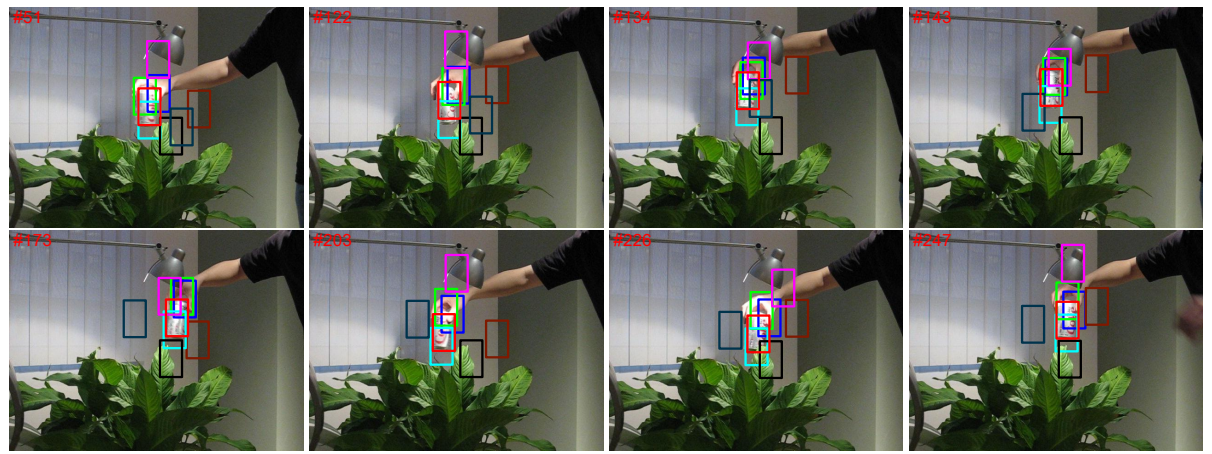

(a) Coke
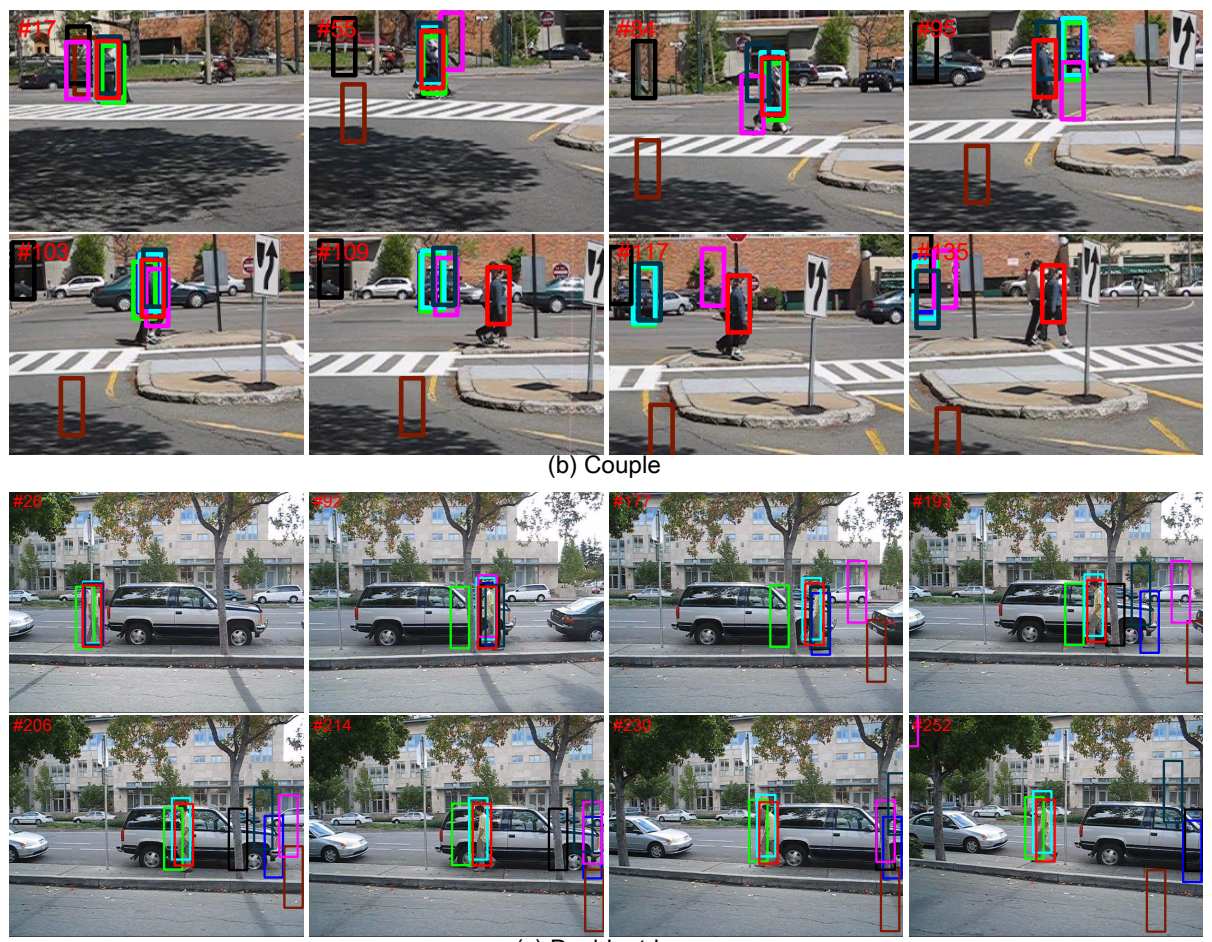

(c) Davidoutdoor

$$
\text { - L1 - CT - MIL - IVT - Frag - WMIL - LOT — Ours }
$$

Figure 7: Sampled tracking results for tested sequences of (a) Coke, (b) Couple and (c) DavidOutdoor. 
completely from drifts to the background at frames $\# 7$, \#17, \#39, \#43, \#56, \#60 and \#68. In the Coke sequence, we can see that our method perform better than other all evaluated algorithms (see all shown frames in Figure 7)(a)). For the Couple sequence, our tracker performs better than the other methods whereas the WMIL, LOT, and MIL algorithms are able to track the objects in some frames. In the Lemming sequence, only the $\mathrm{CT}$ and our method perform well at frame \#550, while the other algorithms fail to track the target objects well. What is more, the Frag method suffers completely from drift in the shown frames, which verifies that the Frag method cannot adaptively adjust these changes, resulting in serious drifts. We can also see that the LOT method can track the object well except that there are few drifts in a couple of frames see frames \#550 and \#1105). Blurry images exist in the Deer sequence (see Figure 8(b)), because a fast motion make it difficult to track the target object. 375 As shown in frames \#56 and \#71 of Figure 8(a), our proposed method can still track the object well compared with other methods.

Background clutters: The trackers are easily confused an object is very similar to its background. Figure 8(b), Figure 6(b), Figure 7(b) and Figure 7)(a) demonstrate the tracking results in the Deer, Couple, Lemming and Stone 380 sequences with background clutters. Figure 7 (a) shows different trackers track a yellow cobblestone located among a lot of similar stones. Thus, it is very difficult to distinguish the object from its background and to keep tracking the object correctly. Comparatively, our method and the IVT exhibit better discriminative ability and outperform other methods at frames \#495 and \#535.

385 The MIL and WMIL trackers completely drift to the background at frames \#426, \#495 and \#535, which verifies that the selected features by the MIL and WMIL trackers are less informative than our method. The Frag tracker has severe drifts at all frames except frames \#55 and \#535 because its template does not update online, making it unable to handle large background clutter. The 390 CT method has severe drifts at frames \#426, \#495 and \#535 because it only uses compressive features and the Bayesian classifier is sensitive to background clutter. 

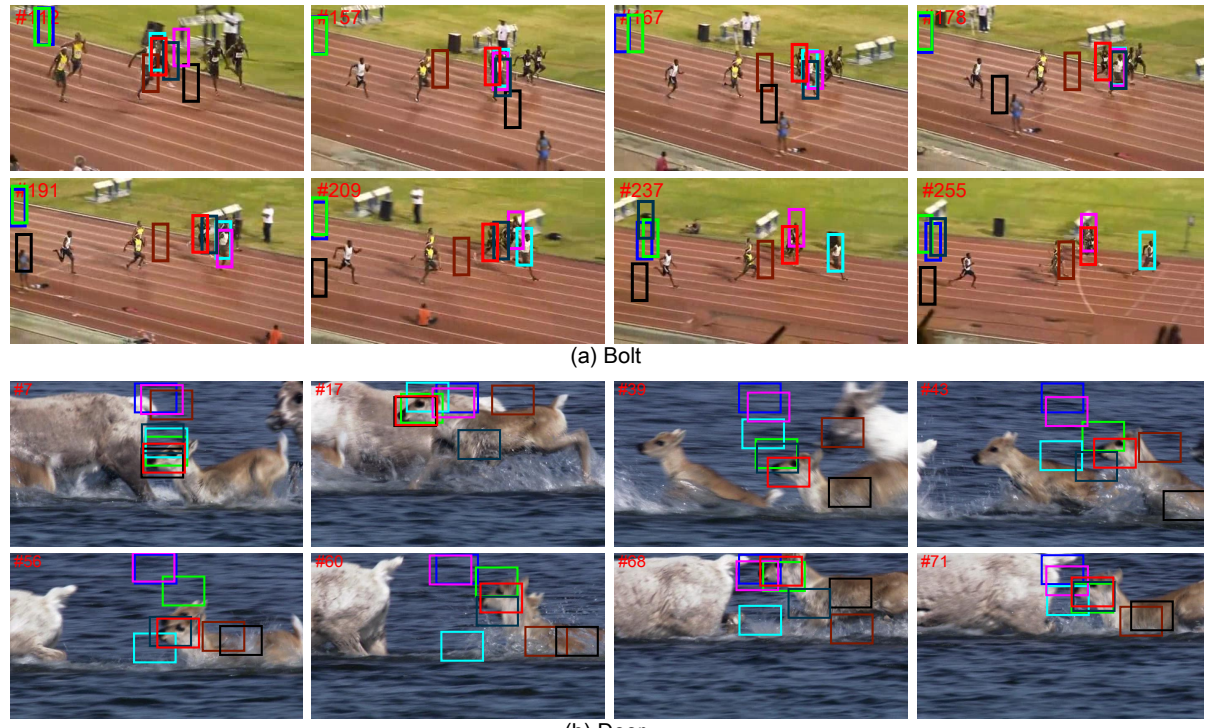

(b) Deer

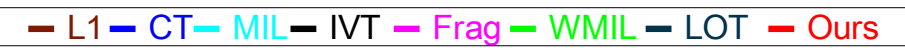

Figure 8: Sampled tracking results for tested sequences of (a) Bolt and (b) Deer.

In the Deer sequence, our method outperforms all other methods in all given frames. In the Lemming sequence, the L1 tracker completely drifts to the background at all given frames, which verifies that sparsity is not useful for tracking. The Frag, IVT, L1 methods suffer from severe drifts at frames \#1105 and \#1126 as shown in Figure 8(b). Meanwhile, the CT performs well too at frames \#1105 and \#1126, but creates a drift at frame \#1096, because it is sensitive to the background clutter and the abrupt motion. In the Couple sequence, the IVT and L1 methods completely fail to track the object at all shown frames, while the MIL, WMIL and CT methods can track well in the first frame. However, they completely fail to track at other frames because they cannot effectively distinguish the object from the background clutters.

\subsection{Comparison our method with other classifiers}

It should be noted that the proposed tracking algorithm is significantly different from other classifiers such as support vector machine method (SVM). 

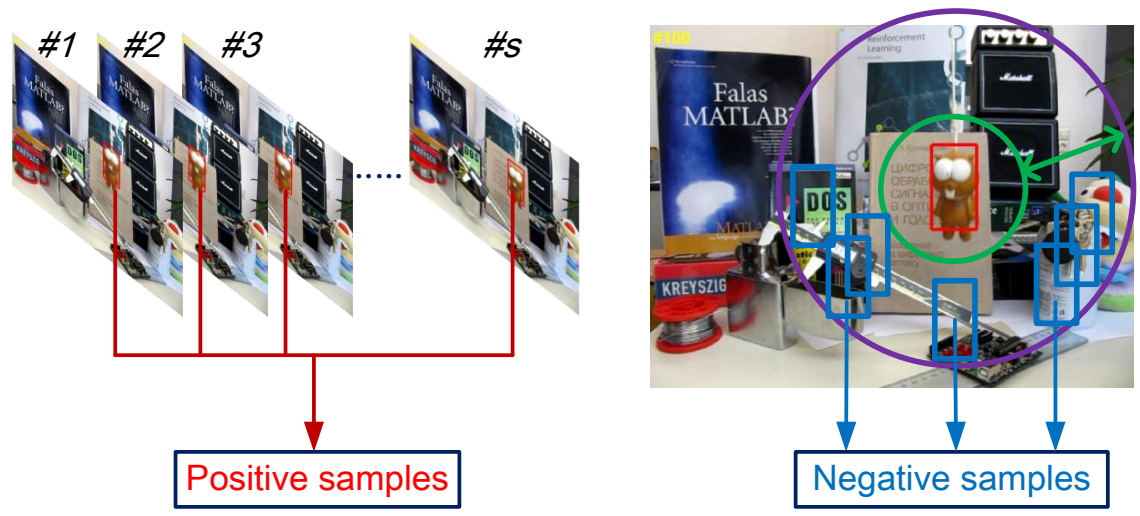

(1) $\operatorname{SVM}(a)$
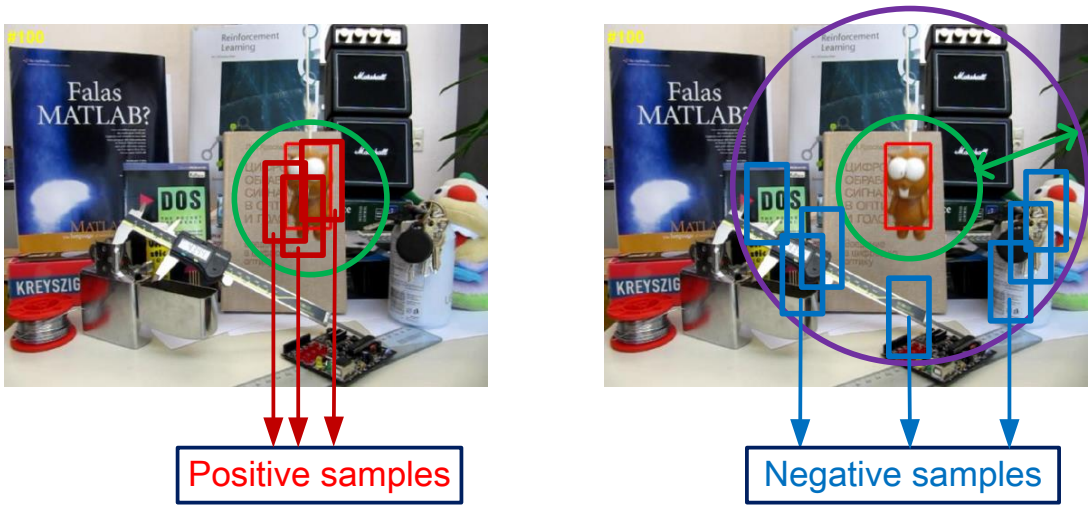

(2) $\operatorname{SVM}(b)$

Figure 9: Two sampling methods using SVM classifier. (1) The tracked results are selected as positive samples, while many image patches are selected away from the current location as negative samples; (2) These image patches around the current location are collected as positive samples, while many image patches are selected away from the current location as negative samples 

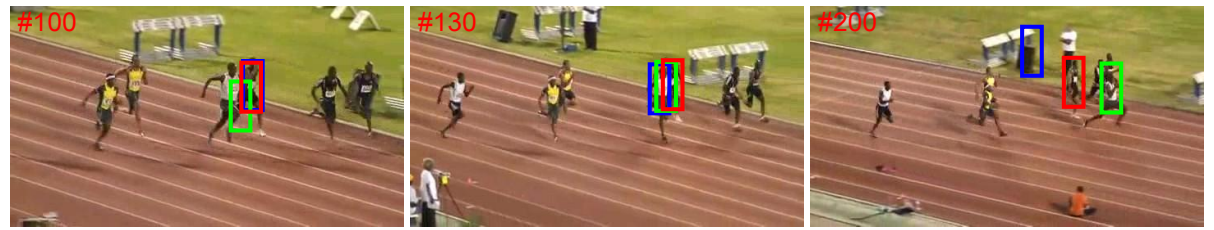

(a) Bolt
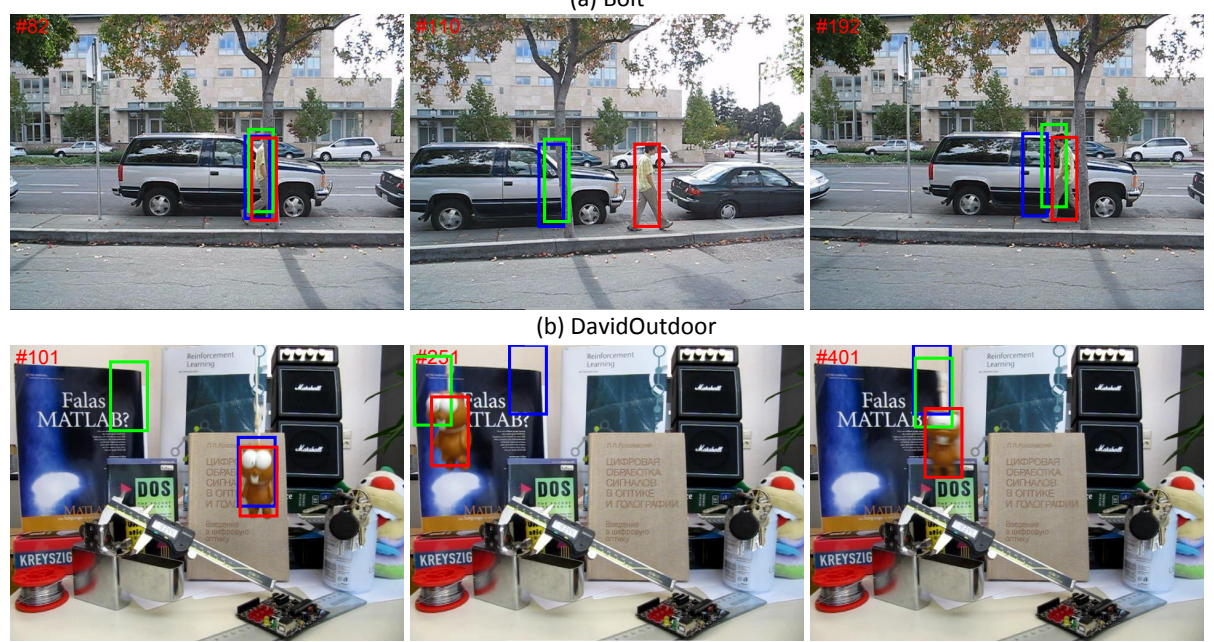

(c) Lemming

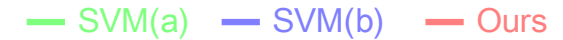

Figure 10: Comparison our tracking method with SVM classifiers.

The outstanding ability of of the manifold learning algorithm is to discover the underlying geometrical structure and the relevance between different data set, while SVM. To verify the performance of our tracker outperforms the performance of using SVM classifier, We construct the two tracking methods using SVM. In Figure 9(1), we assume the locations in the first $t$ frames have been obtained by CT tracker [10, then these tracked results are selected as positive samples, while many image patches are selected away from the current location as negative samples (See Figure 9(1) for details). In Figure 9(2), we collect these image patches around the current location as positive samples, while many image patches are selected away from the current location as negative samples.

In these experiments, we use the Haar feature to represent the object and the dimensionality of the compressive feature is set to 200 . The first $t$ frames 
Table 4: Center location error (CLE) for comparing our method with SVM classifiers.

\begin{tabular}{lccccccc}
\hline Methods & Deer & Stone & Coke & Bolt & Couple & Lemming & DavidOutdoor \\
\hline SVM(a) & 97.1 & 82.4 & 56.5 & 81.8 & 33.4 & 162.1 & 68.7 \\
SVM(b) & 60.2 & 68.9 & 86.1 & 33.4 & 18.9 & 165.6 & 67.9 \\
Ours & 23.0 & 6.4 & 23.9 & 7.6 & 9.3 & 24.3 & 29.5 \\
\hline
\end{tabular}

are tracked by the CT method and $\mathrm{t}$ is set to 30 . Table 4 reports the center location error, where smaller CLE means more accurate tracking results. From Table 4, we can see that our method achieves the best performance compared with SVM classifiers. Figure 10 show the screen captures for some of the video clips. In Bolt sequence, we can see the two SVM methods completely fail to track the target object in the frames \#200 and there are some tracking error in frames \#130 and \#130. In DavidOutdoor and Lemming sequences, our tracker performs better than the other methods.

\subsection{Discussion}

As shown in our experiments, our method can address these factors including abrupt motion, cluttered background, occlusion more effectively. The reason-

$430 \mathrm{~s}$ are as follows. (1) We can extract discriminative features by a very sparse matrix to separate an object well from its background, and the object representation with low-dimensional compressive features can preserve the structure of original image space. (2) The outstanding ability of of the manifold ranking algorithm is to discover the underlying geometrical structure and the relevance between object appearance and candidate samples. (3) Our method combines temporal with spatial context information for tracking, and it is very insensitive to multiple factors. Thus, our tracker can obtain favorable performance.

However, our proposed method may fail when an out-of-plane rotation and an abrupt motion occur in the current sequence (see Figure 11). Figure 11(a) 440 shows an out-of-plane rotation and an abrupt motion after \#75. Our method drifts away the ground truth because the appearance model can not match well 

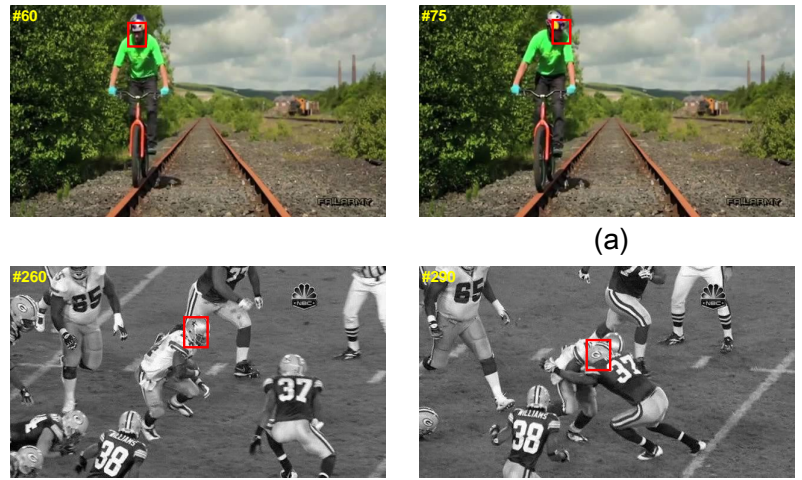

(a)

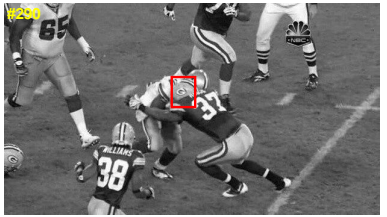

(b)
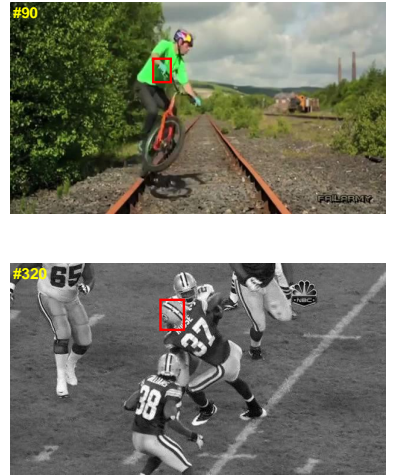

Figure 11: Two failed tracking cases:(a) out of plane rotation and abrupt motion; (b) similar appearance information between object and non-objects.

between the object model and the candidates, and it cannot distinguish the object from the changed background. However, our method is sensitive when there exists a complex background and when there exists similar appearance information between the object and the non-objects in a sequence (Figure 11(b)). Therefore, our method can not distinguish an object from background clutters. Overall, our method performs favorably against the other state-of-the-art tracking methods in the challenge sequences.

\section{Conclusions}

This paper has proposed a novel framework named manifold ranking based visual tracking. The algorithm is initially proposed to rank data along their manifold, which has been widely applied in information retrieval and shown to have excellent performance and feasibility on a variety of data types. In order to address the shortcomings of original manifold ranking from graph reconstruction and heavy computation load, we adopt the efficient manifold ranking algorithm. The ability for efficiently constructing a graph is more applicable for tracking problem. What is more, we adopt non-adaptive random projections to preserve the structure of original image space, and a very sparse measurement matrix has 
been used to efficiently extract compressive features for object representation.

Furthermore, our method exploits temporal and spatial context information

for tracking, which is very insensitive to background clutters and appearance change. Experiments on some challenging video sequences have demonstrated the superiority of our proposed method to seven state-of-the-art ones in accuracy and robustness.

\section{Acknowledgments}

This research is partly supported by NSFC, China (No: 61273258, 61105001), and Ph.D. Programs Foundation of Ministry of Education of China (No.20120073110018).

\section{References}

[1] A. Yilmaz, O. Javed, M. Shah, Object tracking: A survey, Acm computing surveys (CSUR) 38 (4) (2006) 13.

[2] W. Hu, T. Tan, L. Wang, S. Maybank, A survey on visual surveillance of object motion and behaviors, Systems, Man, and Cybernetics, Part C: Applications and Reviews, IEEE Transactions on 34 (3) (2004) 334-352.

[3] A. D. Jepson, D. J. Fleet, T. F. El-Maraghi, Robust online appearance models for visual tracking, Pattern Analysis and Machine Intelligence, IEEE Transactions on 25 (10) (2003) 1296-1311.

[4] A. Adam, E. Rivlin, I. Shimshoni, Robust fragments-based tracking using the integral histogram, in: Computer Vision and Pattern Recognition, 2006 IEEE Computer Society Conference on, Vol. 1, IEEE, 2006, pp. 798-805.

[5] J. Kwon, K. M. Lee, Visual tracking decomposition, in: Computer Vision and Pattern Recognition (CVPR), 2010 IEEE Conference on, IEEE, 2010, pp. 1269-1276. 
[6] X. Mei, H. Ling, Robust visual tracking using 11 minimization, in: Computer Vision, 2009 IEEE 12th International Conference on, IEEE, 2009, pp. 1436-1443.

[7] R. T. Collins, Y. Liu, M. Leordeanu, Online selection of discriminative tracking features, Pattern Analysis and Machine Intelligence, IEEE Transactions on 27 (10) (2005) 1631-1643.

[8] H. Grabner, M. Grabner, H. Bischof, Real-time tracking via on-line boosting., in: BMVC, Vol. 1, 2006, pp. 6-15.

[9] B. Babenko, M.-H. Yang, S. Belongie, Robust object tracking with online multiple instance learning, Pattern Analysis and Machine Intelligence, IEEE Transactions on 33 (8) (2011) 1619-1632.

[10] K. Zhang, L. Zhang, M.-H. Yang, Real-time compressive tracking, in: Computer Vision-ECCV 2012, Springer, 2012, pp. 864-877.

[11] Z. Kalal, J. Matas, K. Mikolajczyk, Pn learning: Bootstrapping binary classifiers by structural constraints, in: Computer Vision and Pattern Recognition (CVPR), 2010 IEEE Conference on, IEEE, 2010, pp. 49-56.

[12] K. Zhang, H. Song, Real-time visual tracking via online weighted multiple instance learning, Pattern Recognition 46 (1) (2013) 397-411.

[13] K. Fu, C. Gong, Y. Qiao, J. Yang, I. Y.-H. Gu, One-class support vector machine-assisted robust tracking, Journal of Electronic Imaging 22 (2) (2013) 023002-023002.

[14] D. A. Ross, J. Lim, R.-S. Lin, M.-H. Yang, Incremental learning for robust visual tracking, International Journal of Computer Vision 77 (1-3) (2008) $125-141$.

[15] T. Bai, Y. Li, Robust visual tracking with structured sparse representation appearance model, Pattern Recognition 45 (6) (2012) 2390-2404. 
[16] H. Grabner, C. Leistner, H. Bischof, Semi-supervised on-line boosting for robust tracking, in: Computer Vision-ECCV 2008, Springer, 2008, pp. $234-247$.

[17] J. He, M. Li, H.-J. Zhang, H. Tong, C. Zhang, Manifold-ranking based image retrieval, in: Proceedings of the 12th annual ACM international conference on Multimedia, ACM, 2004, pp. 9-16.

[18] D. Zhou, J. Weston, A. Gretton, O. Bousquet, B. Schölkopf, Ranking on data manifolds., in: NIPS, Vol. 3, 2003.

[19] B. Xu, J. Bu, C. Chen, D. Cai, X. He, W. Liu, J. Luo, Efficient manifold ranking for image retrieval, in: Proceedings of the 34th international ACM SIGIR conference on Research and development in Information Retrieval, ACM, 2011, pp. 525-534.

[20] T. Zhou, X. He, K. Xe, K. Fu, J. Zhang, J. Yang, Visual tracking via graphbased efficient manifold ranking with low-dimensional compressive features, Multimedia and Expo (ICME), 2014 IEEE International Conference on, IEEE, 2014 (Accepted).

[21] C. Yang, L. Zhang, H. Lu, X. Ruan, M.-H. Yang, Saliency detection via graph-based manifold ranking, in: Computer Vision and Pattern Recognition (CVPR), 2013 IEEE Conference on, IEEE, 2013, pp. 3166-3173.

[22] P. Viola, M. Jones, Rapid object detection using a boosted cascade of simple features, in: Computer Vision and Pattern Recognition, 2001. CVPR 2001. Proceedings of the 2001 IEEE Computer Society Conference on, Vol. 1, IEEE, 2001, pp. 511-518.

[23] D. Achlioptas, Database-friendly random projections: Johnsonlindenstrauss with binary coins, Journal of computer and System Sciences 66 (4) (2003) 671-687.

${ }_{535}$ [24] K. Zhang, H. Song, Real-time visual tracking via online weighted multiple instance learning, Pattern Recognition 46 (1) (2013) 397-411. 
[25] S. Oron, A. Bar-Hillel, D. Levi, S. Avidan, Locally orderless tracking, in: Computer Vision and Pattern Recognition (CVPR), 2012 IEEE Conference on, IEEE, 2012, pp. 1940-1947.

[26] Y. Wu, J. Lim, M.-H. Yang, Online object tracking: A benchmark, in: Computer Vision and Pattern Recognition (CVPR), 2013 IEEE Conference on, IEEE, 2013, pp. 2411-2418.

[27] M. Everingham, L. Van Gool, C. K. Williams, J. Winn, A. Zisserman, The pascal visual object classes (voc) challenge, International journal of $545 \quad$ computer vision 88 (2) (2010) 303-338. 